\title{
Unconscious Emotions in Media Content
}

\author{
Huang-Ming Chang, Leonid Ivonin, and Matthias Rauterberg
}

\section{Contents}

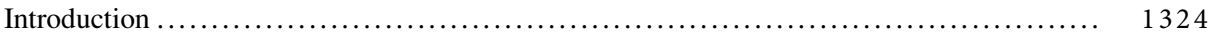

The Unconscious ...................................................... 1326

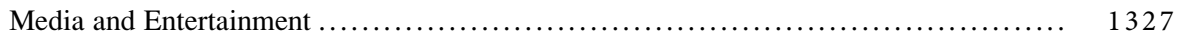

Emotions in Media Content ........................................... 1328

Theories, Models, and Measurement ........................................ 1330

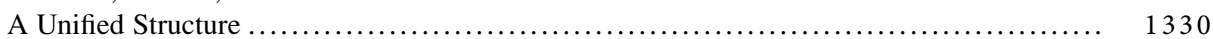

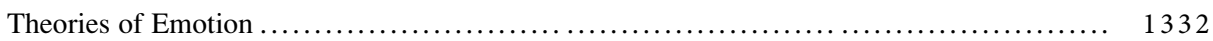

Evolution-Based Theories .............................................. 1332

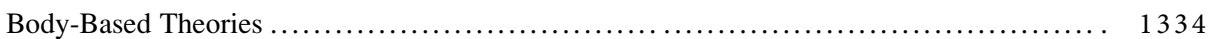

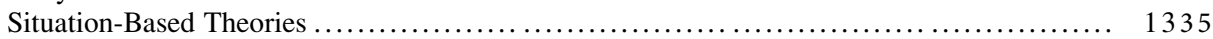

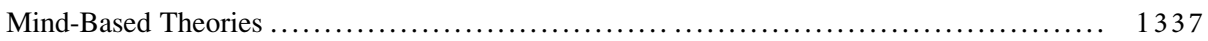

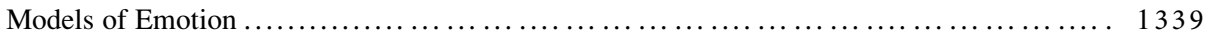

Discrete and Dimensional Models of Emotion ............................... 1339

Building Models with Physiological Signals .................................. 1340

Measures of Emotion .................................................. 1341

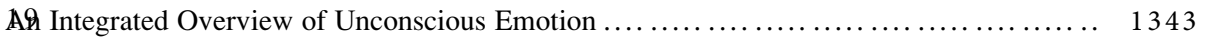

Research into Unconscious Emotions in Media Content ......................... 1345

Mapping Media Experience with the Storyline .................................. 1345

Potential Challenges and Promising Approaches ................................ 1346

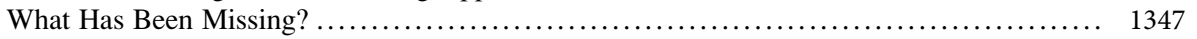

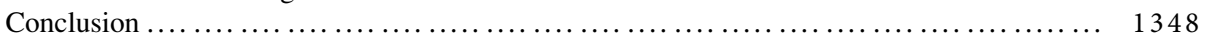

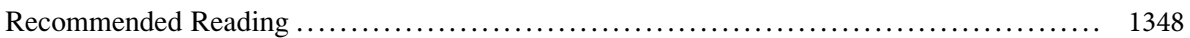

H.-M. Chang $(\bowtie) \bullet$ L. Ivonin $・$ M. Rauterberg

Department of Industrial Design, Eindhoven University of Technology, Eindhoven, The

Netherlands

e-mail: Huang.M.Chang@gmail.com; Leonid.Ivonin@gmail.com; G.W.M.Rauterberg@tue.nl 


\section{Abstract}

Stories and human society are inseparable. Before human history, our ancestors created myths as the oldest form of narratives. Due to the advances of digital media, stories are nowadays communicated in various ways. Yet the structure and the key elements of modern stories still remain the same. This chapter starts with a discussion about the relationship between stories and human society. It is curious that human beings keep being intrigued by stories of similar structures and scenes. A reasonable speculation is that these identical components of stories might induce emotional experiences at an unconscious level. Can emotions be unconscious? While the answer to this question is still open to debate, more and more empirical evidence suggests that humans might have unconscious emotions in certain conditions. In order to provide a holistic theoretical overview focusing on unconscious emotions, a review of contemporary theories, models, and measures of emotion is provided. This review illustrates an integrated overview of research into unconscious emotions and also points out potential challenges and promising approaches that could inform future research on unconscious emotions in media content.

\section{Keywords}

Media content - Unconscious emotion - Emotion theory $\bullet$ Emotion model $\bullet$ Emotion measurement $\bullet$ Review

\section{Introduction}

As part of human nature, people are always interested in exploring the world where they live in. Why does the sun come up from the east? Why are there four seasons? This world is mysterious, not only for our ancestors but also for people in the modern society. Before human beings were capable of justifying a hypothesis with objective evidence (i.e., the scientific revolution), our ancestors formed an imaginative worldview about how this world is built. Myths were thus created to serve the function of media. The relationship between myth and science is a subject as old as that of myth and science themselves. The modern view toward myths varies chronologically by the centuries. In the nineteenth century, myth and science were commonly considered to be opposite and incompatible. In the extreme, people at that time thought of defeating myths by scientific approaches as a sign of a modern society. It was believed that because of the advances of science, this world is becoming less mysterious, so modern people have to abandon myth. On the contrary, in the twentieth century, myth and science were usually taken to be compatible, and therefore myths are once again embraced by the modern society. There is no doubt that the quality of material life substantially relies on science and technology, but it needs to be noted that myths have been enriching the mental life of modern people in many ways. 
Myth is the oldest form of narrative in human history. Since there are no historical records or scientific proof, it is difficult to justify if these mythical narratives are true facts or just merely imaginary stories made up by ancient people. Nevertheless, myths are still influencing modern society and still fascinating to people nowadays. Myths, as a primeval form of living reality, have been woven into people's lives in many different ways. It is usually linked with ritual. Together, they were used to regulate how ancient people were to behave toward nature and construct their basic view of how this world was made. Myths and rituals divide an individual human life into three phases: childhood, adolescence, and retirement. Each of these phases is characterized by corresponding motive complexes: love, power, and death (Rauterberg 2011). In general, there are two ways of interpreting myths: literal and symbolic (Dundes 1988). Literal interpretations look into factual or historical bases for myths, while symbolic interpretation prefers to regard myths as a code requiring some deciphering. In the early nineteenth century, myths were treated as nothing different from fables, inventions, and fictions. Thus, rationalists treated myths as delusions and pure imaginations. From a literal perspective, myths would be merely an unrealistic and unscientific version of history. The greatest value gained from myths would probably be anthropology, rituals, and religions, which mostly aim at finding a reasonable explanation or rational speculation for the reality of ancestral societies. Interestingly, it was found that ancient people tend to present symbols as an instance of association with reality, instead of looking for rational causes (Kirk 1975). It appears that myths were not meant to give a rational explanation toward the reality but to provide a symbolic representation that shows the natural tendency of how ancient people interpret the reality that they lived in.

The nature of myth is different from fictions that people read today. More than just storytelling, some scholars consider a myth as a way of making sense of the physical world (May 1991). A myth is a sacred narrative usually explaining how the world and humankind came to be in its present form. One of the foremost functions of myth is to establish models of behavior. Through its myth, a healthy society gives its people relief from guilt and anxiety and encourages humans to discover true values in their lives. Rather than merely imaginary stories, myth is a narrative resurrection of a primeval reality. "It is a living reality, believed to have once happened in primeval times, and continuing ever since to influence the world and human destinies" (Malinowski 1954). Indeed, it is surprising that even though the content of myths seems unrealistic and irrational, myths are still manifested in modern media content, such as artwork, music, and movies. Numerous ancient myths are being reedited as a modern version and remade into various forms of digital media. Although the content of the myths might change overtime, the skeleton of the stories still remains intact. The human society grows with myth, while myth manifests the thinking and behavioral pattern of the people within the society. This circular relationship has been continuously working along with human history, and, nowadays, many traces of myth still can be seen in the modern society. 


\section{The Unconscious}

Some cultural rituals and customs can be traced back to historical events, but others could only count on myths, fairy tales, and legends. Although these fictional narratives cannot be proved to be true, they lie at the heart of people and form the knowledge that guides people how to see things and act in the society. Fictional narratives have been an important medium for the growth of culture and society. They provide a deep and immersive simulative experience of social interactions. Furthermore, it is believed that people's thoughts and behaviors are deeply and implicitly influenced by their social and cultural context. In recent years, psychologists start to investigate how people could adapt themselves into a given social situation. The results suggest that this adaption not necessarily be a conscious action but can be unconscious (Bargh and Morsella 2008). Indeed, many of our decisions and behaviors are irrational and driven by the unconscious. While most people think irrationality is harmful for decision-making, empirical studies have revealed the merits of utilizing the unconscious to achieve better results in a complex decision-making task (Dijksterhuis 2004). Sometimes people make decisions based on preferences instead of rational reasoning. In fact, it is an inborn capability of humans to look for better conditions in terms of physical and mental well-being. While a cognitive task is beyond the capability of rational thinking, the intuitive, unconscious, irrational thinking might take over and solve the problem with "gut feelings" (Gigerenzer 2007).

The interests in human unconscious were first revealed by Freud (1922). After being quiet for a long time, research on the unconscious regains the attention of the science community in recent years. Some psychologists strictly hold a consciouscentric model of the human mind, considering the unconscious as a low-level mental processing system that handles subliminal-strength stimulation from the environment, while the conscious processes still play the primary role in causing human judgment and behavior (Bargh and Morsella 2008). Nevertheless, other scientists uphold the priority of the unconscious and suggest that the unconscious processing is not limited at the low-level perception but can also handle high-level mental activities, such as decision-making, goal setting, attitude, and emotion regulation. The distinction between conscious reasoning and unconscious thinking is significant. According to Kahneman (2003), there are three cognitive systems of the human mind: perception, intuition, and reasoning. Perception and intuition form an experiential system that processes the incoming information in real time and generates corresponding reactions automatically. Only part of the incoming information would pass the filter of attention and enter into the rational system for logical reasoning.

In addition to the cognitive account for the unconscious, psychologists also take into account the impact from the social and cultural context. People who live in a common social context would automatically and implicitly develop collective intentionality - a kind of knowledge that is formed and shared within a group of people (Barrett 2012). This knowledge provides a basic reference for people to understand the external world and thus generate emotion, memory, and other 
mental content. When being part of a society, people tend to adapt themselves into the given social context, and this adaption might take place outside of conscious awareness. While it seems to be a valid assumption, it is still unclear how this collective intentionality is achieved within a large group of people, e.g., a society. Human communication is complicated and versatile. Besides the literal interpretation toward the use of languages, human beings tend to utilize a higher level of skill to communicate abstract notions. Some sociologists propose that all kinds of human communication are symbolic (Manis and Meltzer 1978). Their fundamental premise is that humans do not directly react to the ontological-existing reality but respond to their understanding of this reality. It is a natural tendency for humans to express abstract notions in an associative and symbolic manner. Symbolic meaning appears to be an important layer that lies between the human mind and the physical world. The meaning-making process is automatic, spontaneous, and irreversible. Emotion, as one of the essential psychological phenomena, allows people to respond to the meaning they perceived from the world and thus shapes their behaviors.

\section{Media and Entertainment}

While the pure science looks for precision and rationality, the application domain of entertainment aims to create emotionally intense, rich-in-meaning media content. Entertainment is part of people's daily lives. The history of entertainment can be traced back to the ancient times. It is not only because humans need to rest from work but also due to the desire for enriching the mental and spiritual life. The essence of entertainment is versatile. Rather than a means for merely having fun, entertainment plays the role in maintaining mental sustainability and facilitating social transformation. It can also be a kind of media that delivers cultural experiences at an unconscious level. With the advances of media technology, the modern form of entertainment offers tremendous potential for making positive effects on human behaviors in various ways. In this regard, it is needed to consider how entertainment gradually changes with the growth of human's society and how future entertainment technologies might in turn make impact to people's lives.

Since the 1980s, the emergence of computational technology and the advances of multimedia communication have considerably changed people's lives. Today, multimedia systems have widely spread and permeated to people's daily lives. Mobile technology and Internet enhance the accessibility of media content, and communication becomes richer in terms of content. Entertainment, as an important category of media content, becomes one of the driving forces that thrust the development of new types of multimedia systems. Media technology has broadened the bandwidth of communication for transmitting entertaining content to people by using multimodal channels. However, the content of the entertainment is equally important and worth studying. Myths, as the media content in ancient times, were spread through word of mouth and symbolically represented as artwork. These mythical stories were not lost along with the growth of human society and still are 
being manifested in various media forms, such as video games and movies. This curious phenomenon has demonstrated the universal human capacity to classify, to codify, and to communicate their experiences symbolically in different time scales.

There are two kinds of interpretations toward media content: rational and emotional. With regard to functional purposes, communication needs to be precise and logical, so the media content has to be descriptive and concrete in order to achieve a clear understanding of the enclosed message. On the contrary, media content for entertaining purposes particularly emphasizes the emotional aspect of the communication. While the rational interpretation of media content looks for simplicity, emotional interpretation of media content is abstract, dynamic, symbolic, and experiential. This categorization also resonates with the distinction between the two cognitive systems of the human mind. The emotional aspect of communication encompasses a considerable amount of information that would be handled by higher-dimension mental process: the unconscious. With these two interpretations, a pure, holistic experience thus can be delivered in a more comprehensive manner - a rich combination of communication channels that allows conscious and unconscious information flow freely.

\section{Emotions in Media Content}

In this chapter, it is intended to explore the relationship between two main subjects: emotion and media content. Media can be referred to any means of information communication. While the form of the media is progressing with the advances of technology, it is needed to concentrate on how media content becomes meaningful to the audience. Meaning, on the other hand, is becoming an important topic in psychological research on emotion. In the early years, it was assumed that emotion was directly caused by external stimuli in the environment, such as natural threats (snakes or earthquakes) cause the feeling of fear. Today, psychologists have developed more comprehensive explanations for the activation of emotion. Mainstream psychological theories suggest that the meaning of the given situation plays an important role in activating emotions (Arnold 1960). For example, natural threats are interpreted as a cause of death, and this interpretation thus activates the feeling of fear. In this regard, the symbolic meaning of media content plays a key role in bridging the gap between emotion and media content.

Narratives have been the primary media content for transmission of emotional experience since early times of the mankind. In essence, narratives are a kind of virtual reality, which pulls the reader into a fictional world. As it is mentioned earlier, fictional narratives usually reflect the worldview of the modern time. Different from history, which is meant for documenting the true fact, the function of narratives is to render the social atmosphere in an implicit manner and deliver rich emotional experiences along with the interaction among the characters. Most people tend to enjoy the mediated emotional experience rather than rationally analyze the logic of the content. Just as children love often-told bedtime stories, sometimes adults enjoy watching classic movies over and over again and never tire 
of them. Narratives can induce profoundly emotional experience. However, very few studies have addressed this topic within psychology probably due to the misconception that narratives are mere made-up illusions (Oatley 1999). Recent years have seen some researchers advocating the importance of narratives in psychology. They suggest that narratives operate as a means of simulating social and emotional experience (Oatley 1999; Mar and Oatley 2008). The evoked emotion may even influence the viewer's daily life after viewing. Although most of these studies particularly focus on literacy narratives, they still shed some light on narratives in various media types, such as paintings, comics, theater plays, and movies. If media are considered as an instrument for transmitting emotional experience, narratives can thus be defined as a design pattern of media content. In this regard, exploring the relationship between the pattern of narratives and its corresponding emotional responses appears to be a promising research direction.

Emotion is an important aspect of media experience, and it has been of great interest to the design community. Studies on emotional aspect of design usually adopt psychological theories of emotion as their theoretical basis. However, the mainstream psychological studies solely focus on utilitarian emotions (Scherer 2005). These types of emotions can be considered utilitarian in the sense of facilitating our adaptation to events that have important consequences for our well-being, such as fear triggers fleeing from danger. However, emotions in media content do not seem to have survival values for maintaining physical sustainability. This view appears to be limited and cannot explain emotional experiences in the context of media design, i.e., why people enjoy watching movies while movies do not bring any survival values? While it has been well researched that emotion has biological and social functions, other aspects of emotion remain mysterious and intriguing. Moreover, the question of whether emotions can be unconscious draws great attention in recent years. Psychologists hold various theoretical positions and use different measures to approach this hypothetical phenomenon. According to some accounts, emotion cannot, by definition, be unconscious (Izard 2009), while other accounts hold that emotions and other psychological phenomena can be unconscious (Kihlstrom 2008). For further justification of unconscious emotion, scientists are seeking experimental evidence to support their belief that emotion may be unconscious, at least under certain conditions. Today, the issue of unconscious emotion is still open to debate; it is difficult to judge whether the phenomenon exists or is just an anecdote. Notwithstanding the uncertainty surrounding them, the early findings have encouraged researchers to reassess existing conceptual and operational definitions of emotion and look for ways to accommodate the new evidence within their theoretical framework. 


\section{Theories, Models, and Measurement}

Putting all emotion-related literature on a continuum between theory and practice, the main body of emotion research can be organized into three building blocks: theories, models, and measurement. In most cases, researchers confuse theories with models and refer to both of them as theories, but they take entirely different epistemological positions. Theories of emotion treat emotion as a natural phenomenon to be explained. Thus, they elaborate the ontology and functionality of emotions and focus on its conceptual definition. On the other hand, models of emotion are built to demonstrate the taxonomy of emotion by conceptualizing all kinds of emotions in a meaningful way. These models of emotion decompose emotions into meaningful and manipulatable parameters so that they can be used for practical purposes, such as affective computing. In order to provide evidence relevant to hypotheses derived from specific theories and models, various measures of emotion have been developed. Operationalizing the concept of emotion in this way has allowed researchers to move beyond biology and philosophy and assert the credibility of psychology as a science. However, the measurement of emotion is challenging because it is difficult to ensure that "the measure does index the emotion it is assumed to be related to." The validity of measurement is often a concern in psychology. Biased measurement methods significantly influence the results of experiments, and the interpretation of data can vary depending on the theoretical approach adopted.

When considering a new hypothesis such as the existence of unconscious emotion, researchers need to pay attention to the epistemic tradition within which theory, model, and measures are applied in order to decide whether the evidence is sufficient to support or reject the hypothesis. It is required to include at least two of the building blocks to fulfill the construct validity of research. Current empirical evidence for the existence of unconscious emotion is derived from research based on different assumptions and is insufficient to answer all the objections that have been raised. In order to understand clearly how the empirical data should be interpreted in terms of the various current theoretical perspectives, it is necessary to review carefully the various theoretical arguments put forward by proponents of the concept of unconscious emotion.

\section{A Unified Structure}

In order to review various theories of emotion, it is necessary to develop a unified descriptive structure to represent the process of emotion activation (see Fig. 1). The basic process by which an emotion emerges can be broken down into three stages: elicitation, emotional state, and reaction (Rottenberg et al. 2007). Usually the term "emotion" refers only to the second stage, which is the emotional state of the individual at any given moment. However, the cause of the emotion (i.e., elicitation) and how this emotion influences the individual (i.e., the individual's reaction to the emotion) are equally important; if these components of emotion are 


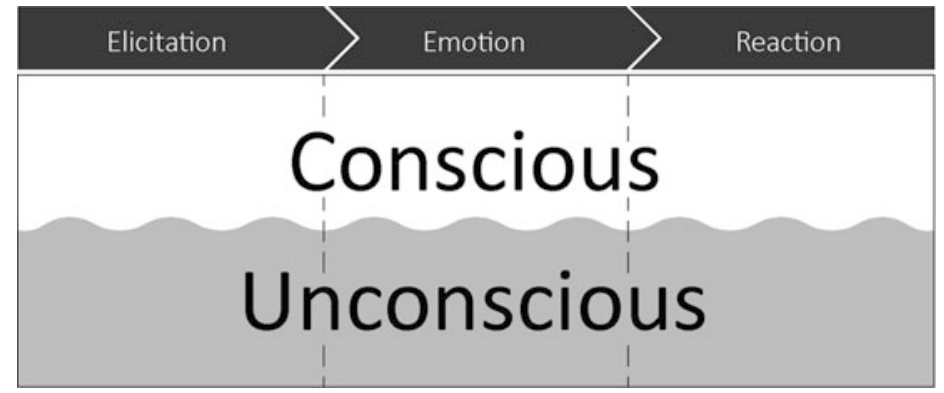

Fig. 1 The conceptual structure of emotion on which the review is based

neglected, the discussion becomes purely philosophical and ceases to be grounded in empirical data. The elicitation of emotion can take almost any form; any information that comes from the external world may elicit an emotion. The concept is not restricted to specific objects or events that experimental psychologists usually refer to as stimuli, but may include social or cultural contexts. Similarly, when it is referred to the reaction to an emotion, the concept covers not only observable physical activities (e.g., behaviors, facial expressions, and physiological responses) but also mental activities (e.g., thoughts, preferences, attitudes, and decisionmaking). This three-stage structuring of emotion as a process has been used to ensure the consistency and clarity of the rest of the review.

To integrate unconscious emotion into this structure, a line must be drawn at each stage to indicate the border of the conscious; the area beneath this border then represents the unconscious. In order to define the border between the conscious and the unconscious, it is necessary to a more complex question: what does "unconscious" mean? Cognitive psychologists usually equate "the unconscious" with subliminal information processing (Kihlstrom et al. 2000). By this definition, unconscious processing deals only with low-intensity, unsophisticated mental activities below the threshold of perception, such as subliminal perception. Social psychologists use a broader definition of the unconscious mind (Bargh and Morsella 2008). They consider the unconscious to be those mental processes that the individual cannot access through introspection (Wilson 2003). In order to accommodate most of the theories, the later definition is used as the criterion of conscious emotional process. The way in which this criterion defines the border between the conscious and unconscious at each stage is as follows: At the first stage, emotion elicitation, the border is defined in terms of the degree to which the individual is able introspectively to recognize stimulus information. At the second stage, the momentary emotional state, the differentiation between the conscious and the unconscious depends on whether the individual can recognize introspectively the emotion that is being experienced. At the third stage, emotional reaction, the border is defined by the degree to which the individual is aware introspectively of the 
changes in his or her own behavior, thought, or bodily sensations. It should be noted that these three stages can be either conscious or unconscious, respectively, to constitute the whole process, i.e., a conscious activity at one stage may be related to unconscious activity at another stage and vice versa. For example, a stimulus that is not consciously recognized might elicit a conscious emotion; similarly, an unconscious emotion may produce a conscious reaction. In the remaining sections of this review, the mental content at each stage will be specified in terms of this structure according to the assumptions of the theory under discussion.

\section{Theories of Emotion}

Many theories of emotion suggest that an emotion emerges when an individual makes sense of something as being in some way related to or caused by the situation (Gendron and Barrett 2009). Rather than thinking of emotion as a state, it has been suggested that emotion should be considered as a process of conceptualizing the world (Barrett 2012). While the human mind has a variety of cognitive functions such perception, learning, and memory, there are higher mental mechanism operating beyond cognition, such as emotion (Kihlstrom 2008). Most psychologists agree that the activation of emotion is effortless and automatic, not controlled (Kihlstrom 2008; Barrett 2012). However, the key question is: what is the source of emotion activation? Contemporary theories of emotion can be categorized into four different perspectives: evolution based, body based, situation based, and mind based.

\section{Evolution-Based Theories}

The functional-evolutionary perspective defined emotion in a teleological fashion by stating that emotions are evolved phenomena with important survival functions that have been selected for because they have solved certain problems that humans have faced as a species. As results of natural selection, to some degree all humans should share a common pattern of emotional expression and the innate capability to interpret each other's emotions, especially through facial expression. This branch of theories holds a biological view and asserts that emotions are natural kinds (Barrett 2006a). It assumes that emotions are universally manifested by humans, selected for over the course of evolution and biologically primitive; the fundamental elements of emotional life are thus conceived as an ancestral heritage. In the view of the modern evolutionary theorists, basic emotions are dedicated neural programs or circuits that are hardwired into the brain (Lang 2010); this implies that all emotional reactions are survival-related, preprogrammed physiological reactions and physical behaviors.

Putting this theoretical view into the unified structure, the sources of emotion activation appear to be utilitarian functions with survival value (see Fig. 2). The brain circuits are responsible for making sense of the external situation and generate 


\begin{tabular}{|c|c|c|c|}
\hline Evolutionary & Elicitation & Emotion & Reaction \\
\hline CONSCIOUS & $\begin{array}{l}\text { Presenting stimuli with a } \\
\text { fixed duration }\end{array}$ & $\begin{array}{l}\text { Biologically hardwired } \\
\text { utilitarian functions }\end{array}$ & $\begin{array}{l}\text { - Facial expression } \\
\text { - Physiological signals }\end{array}$ \\
\hline $\begin{array}{l}\text { Perceptions } \\
\text { available to }\end{array}$ & & & • Self-report \\
\hline introspection & $\begin{array}{l}\text { Subliminal mere exposure } \\
\text { - Natural threats } \\
\text { - Smiling human faces } \\
\text { - Frowning human faces }\end{array}$ & $\begin{array}{l}\text { Emotions include: } \\
\text { - Happiness } \\
\text { - Fear } \\
\text { - Anger }\end{array}$ & $\begin{array}{l}\text { Unintentional changes in } \\
\text { - Behavior } \\
\text { - Preference }\end{array}$ \\
\hline
\end{tabular}

Fig. 2 Evolution-based perspective

emotions accordingly to drive survival mechanisms. For example, external threats trigger the relevant brain circuits and thus generate the emotions of fear or anger, which in turn produces an impulse to flee or fight. The direct causation of behavior is the primary function of emotion. Since emotions are defined as natural kinds and the brain circuits are hardwired, emotion activation itself is more like an instinct. Such instincts could be accurately defined as fixed action tendencies corresponding to utilitarian functions, meaning that most emotional reactions are observable as spontaneous, homologous facial expressions and bodily reactions. Although the contemporary evolutionary perspective considers emotions to be related to social context, facial expressions were considered as a natural adaptation to the social environment, and social events were conceived as survival challenges from an evolutionary perspective (Öhman 1986). In evolutionary emotion research, a common approach to emotion elicitation is the presentation of affective pictures, specifically images of facial expressions and natural threats. As for measuring emotional reactions, the evolutionary perspective holds that all emotions can be manifested through facial expression and behaviors as it assumes that emotions are preprogrammed and universally recognized.

From the evolution-based perspective, the activation of basic emotional systems is to some extent independent of conscious awareness of the current situation. With regard to the unified structure, since emotion activation is considered biologically coded and hardwired in the brain circuits, the border between conscious emotion and unconscious emotions can only be the threshold of perception: conscious emotions are emotions triggered by stimuli that are perceived consciously, while unconscious emotions are emotions induced by stimuli that are exposed below the threshold of perception. This has led to considerable research into subliminal processing of affective stimuli. Researchers who take an evolutionary perspective argue that some mental activities can occur without being available for introspection, such as implicit perception and implicit cognition (Kihlstrom 2009). This claim was substantially supported and implicit processing characterized by empirical studies on the mere exposure effect or subliminally priming effect (Zemack-Rugar et al. 2007; Bornemann et al. 2012). Emotional reactions related 
to subliminal priming are not measured directly from facial expression or physical behaviors as in studies on conscious emotions; rather they are assessed from indirect measures such as changes in preferences and changes in behavior when performing a specific task which is held to be significantly related to affect (Winkielman et al. 2005).

\section{Body-Based Theories}

Contrary to earlier psychologists who believed that emotions were mental events that caused physical changes in the body, some researchers asserted that emotion emerges immediately after one perceives an internal physical sensation that is triggered automatically and adaptively by the external environment (James 1884). This counterintuitive proposition led to a new approach to research on emotion but also generated considerable theoretical debate specifically about the temporal relationship between the physical sensation and the emotional experience. Yet, it is still valid to assume that emotion emerges through making the internal physical sensation meaningful.

The theories of psychological constructionists consider emotion to be an act of sensemaking directed by the internal sensory or affective state (Gendron and Barrett 2009). In contrast to the basic emotion theorists, psychological constructionists claim that emotions are not biologically given but are constructed through the process of making sense of physical changes arise in the body (Barrett 2006b). The basis of the sensemaking process is also not a hardwired instinct; rather it is socially shared conceptual knowledge (Barrett 2012). The psychological constructionist view considers that emotions are socially related but cannot be reduced to mere social situations, as it holds that the presence of an individual mind is necessary to the sensemaking process. Sensemaking is the core concept of psychological constructionist approach to emotion, as Barrett states: "Human brains categorize continuously, effortlessly, and relentlessly...Via the process of categorization, the brain transforms only some sensory stimulation into information. . . To categorize something is to render it meaningful" (Barrett 2009).

Damasio (1996) proposed a similar hypothesis, namely, that conscious emotions are actually feelings - emotions can be consciously felt through perception of the bodily sensation. Similar to the basic emotion theory, he defined emotions as biological life-regulation phenomena that are reflections of cognitive actions in human bodies toward a given situation. According to Damasio, what people perceive as emotional feelings are composite perceptions of these biological phenomena; in other words, feelings are images of emotions, rather than emotions themselves. Feelings and emotions are therefore not always identical, and not all emotions can be perceived as feelings. Some empirical evidence on significant changes in physiological data has supported this hypothesis. These data occur when an individual is performing certain decision-making tasks. Changes in an individual's physiological signals - somatic markers is Damasio's term - indicate when a risky choice is being made although the individual is unaware of them (Bechara 


\begin{tabular}{|c|c|c|c|}
\hline Body-based & Elicitation & Emotion & Reaction \\
\hline CONSCIOUS & $\begin{array}{l}\text { Stimuli or tasks with } \\
\text { contextual information }\end{array}$ & $\begin{array}{l}\text { Feelings; perceptions of } \\
\text { bodily sensations }\end{array}$ & $\begin{array}{l}\text { Perceptible } \\
\text { physiological signals }\end{array}$ \\
\hline $\begin{array}{l}\text { Bodily sensations } \\
\text { available to }\end{array}$ & & & \\
\hline UNCONSCIOUS & None & $\begin{array}{l}\text { Biological life-regulation } \\
\text { phenomena by cognitive } \\
\text { actions in our bodies }\end{array}$ & $\begin{array}{l}\text { Imperceptible } \\
\text { physiological signals }\end{array}$ \\
\hline
\end{tabular}

Fig. 3 Body-based perspective

et al. 2005). He has put forward a promising theoretical perspective that is complementary to the psychological constructionist view of emotion in that the bodily sensation is the target of the sensemaking process.

Based on the above theories, being able to make sense of physical sensations in introspection appears to be the primary difference between conscious and unconscious emotions. As the psychological constructionists suggest, the sensemaking process relies on a connection to the situation in which the emotion emerges. In other words, when a mismatch or loss of connection occurs during the sensemaking process, the emotion is unavailable for introspection and thus becomes unconscious. Studies of emotion elicitation from the body-based perspective usually use stimuli with rich contextual information or simulated scenarios, such as images, emotion words, and simulated games, but there have been no investigations using stimuli that are unavailable for introspection (see Fig. 3). To track the emotional reaction, measures of physiological signals and brain states are frequently used, because physiological responses are at the heart of the body-based perspective. Some of the physiological changes in the autonomic nervous system (ANS) are available for attentive introspection, such as heart rate and respiration rate, but other changes such as changes in brain states cannot be detected without biometric equipment.

\section{Situation-Based Theories}

The contemporary situation-based perspective is embodied in the appraisal theory proposed by Arnold (1960). Arnold used the term "appraisal" to describe an essential cognitive mechanism: a given situation is evaluated, and the results of this evaluation then cause emotion. Frijda (1988) described appraisals as assessments of situational meaning: "Input some event with its particular meaning; out comes an emotion of a particular kind." Although appraisals are necessarily intentional, their meaning analyses are automatic cognitive mechanisms specifically dedicated to emotion, and they need not be available to awareness. Social constructionists also take a situation-based perspective but one that differs from that of the cognitive theories of emotion. Rather than looking inwards, social constructionists 
treat emotions as cultural products whose meaning is based on learned social rules (Averill 1980). Social constructionists emphasize the social functions of emotion and how social contexts cause one's emotional state. For them, cultures provide the content of the appraisals that form the basis of emotions, which are constructed within a culture to serve specific social purposes. Unlike the psychological constructionists who hold that emotions are socially related but not socially specific, social constructionists hold that culture plays a central role in the organization of emotions; they consider that emotions can be reduced to the social situation in which they occur (Gendron and Barrett 2009). Rather than considering emotion as an entity, social constructionists define emotion as a dynamic process that emerges while an individual is interacting with others within a given social context. They believe that emotions have a social function and that to understand emotions, one has to consider what a specific emotion accomplishes socially (Averill 1980). This is the fundamental distinction between these two constructionist views: for social constructionists, the target of the sensemaking process is the external social situation, whereas psychological constructionists regard bodily sensations as the target of the sensemaking process.

Taking a similar approach to appraisal theory, some social psychologists argued that emotion might also be inaccessible to the conscious. The concept of the adaptive unconscious (Wilson 2003) was put forward. These authors regarded the sensemaking process as an inferential or self-attribution process by which an individual makes sense of a given situation in terms of his or her bodily sensations. Making inferences about the world is not always rational and intellectual; it may be intuitive, effortless, and adaptive (Gigerenzer 2007). This inferential process can take place either consciously or unconsciously and cause conscious or unconscious emotion accordingly. Surprisingly, some empirical results have revealed that there can be differences between conscious and unconscious emotions related to a given situation (Schachter and Singer 1962). "The conscious system is quite sensitive to personal and cultural prescriptions about how one is supposed to feel... People might assume that their feelings conform to these prescriptions and fail to notice instances in which they do not" (Wilson 2004). Although physical sensations are an important reference for the sensemaking process, the target of it appears to be the situation that the individual finds him- or herself.

According to the situation-based theories discussed above, the distinction between conscious and unconscious emotions is based on whether an individual is able consciously to identify the connection between his or her emotion and the given situation (see Fig. 4). In terms of the unified structure in this review, the emotion elicitation process relies on the external situation, more specifically a social circumstance. As for the emotional reaction, like the evolutionary-based research on emotions, research in the situation-based tradition usually utilizes self-reports of preferences or decision-making tasks to capture conscious emotional responses, while physical behaviors are used as an index of unconscious emotion. This is probably because researchers with a situation-based perspective tend to use connections with the external world as the criterion for distinguishing conscious and unconscious emotion. 


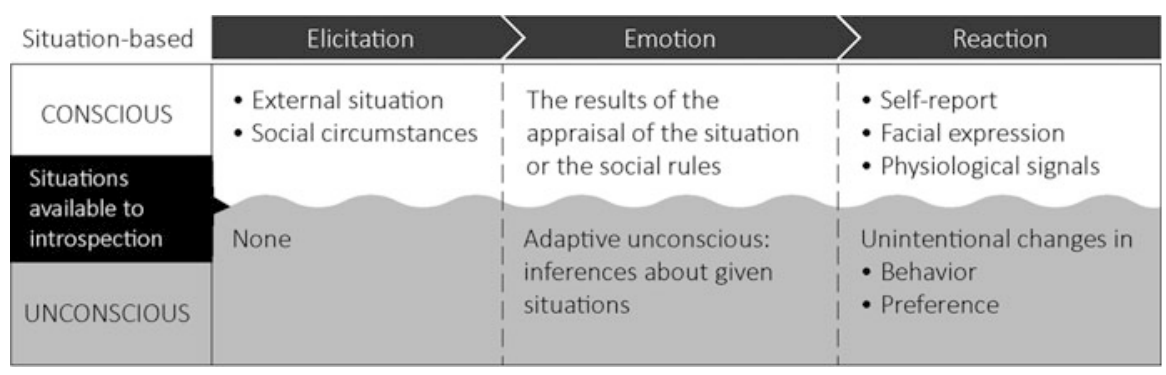

Fig. 4 Situation-based perspective

\section{Mind-Based Theories}

Social psychologists place focus on groups and tend to look externally, to the situation for explanation, whereas the psychoanalytic tradition focuses on individuals and uses internal data to explain inward subjective experience (Chancer 2013). The psychoanalytic tradition stands in opposition to mainstream psychological research; rather than trying to deconstruct emotions, it tends to approach human mind as a whole. In psychoanalytic theories, the unconscious mind is the primary source of psychological content, which is deeper than the conscious mind. Rather than looking for connections to the body or the environment, psychoanalytic theories seek for psychological meanings within the deeper levels of the human mind. The importance of the unconscious was first advocated by Freud (1922); in Freud's original model, the metaphor of the dark cave was applied to the human mind. He argued that the unconscious was a complex dynamic that suppressed primal desires, and these desires can only be represented as anxiety. Since mental content (memories, thoughts, and emotions) in the unconscious was assumed to be inaccessible to conscious introspection, Freud likened psychoanalysis to an archeological dig in which the psychological content of the unconscious would be uncovered (Wilson 2004). Freud assumed that emotions were the outlets for instinctual drives and that emotions emerged when the expression of these instinctual drives was blocked (Freud 1922). Emotions can be felt at the surface of the conscious; however, the cause of these emotions remains buried deep in the unconscious and unreachable for introspection.

The Freudian concept of the unconscious, although it is well known outside psychological science, has not been supported by empirical studies, but it continues to provide inspiration for research in the psychoanalytic tradition. In addition to Freud's concept of the individual unconscious, Jung (1959) proposed an unconscious specifically focused on the psychological content that was common to all human minds. Jung's interpretation of observational data from his patients was that there is a universal pattern to the psychological content of the mind that can only be expressed through symbolic content. Jung argued further that the universal pattern in the unconscious mind contained some contents and modes of behavior that are 


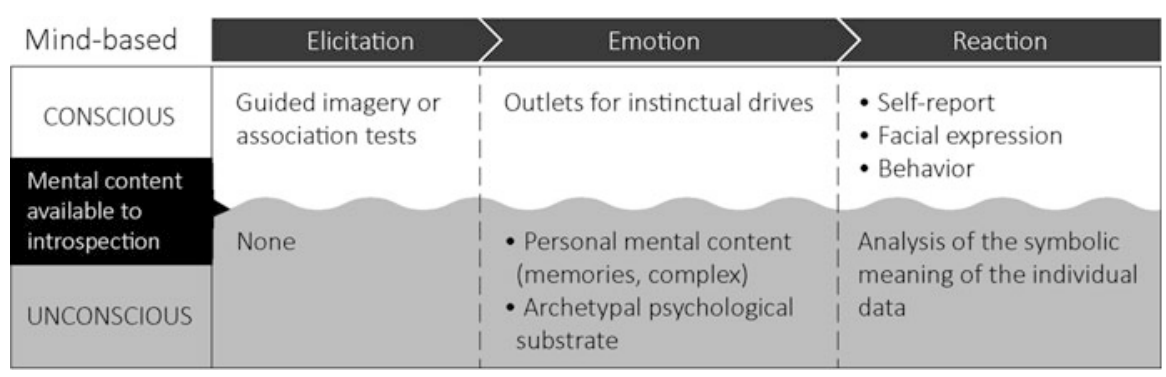

Fig. 5 Mind-based perspective

identical in all human beings and thus constituted the common psychological substrate of a universal nature that is present in every individual. All human beings, sharing essentially the same biological equipment (e.g., the brain and the central nervous system), would show a tendency to perceive common meanings embodied in symbolic content at an unconscious level. Jung's theory has been used for analyzing media content, especially movies (Chang et al. 2013). Although psychoanalysis has often been criticized for the unfalsifiability of its hypotheses because it relies largely on the interpretation of the symbolic meaning of individual data, there has recently been new interest in psychoanalytic theories in neuroscience; surprisingly, new evidence has been found in neuroscience to support some psychoanalytic hypotheses (Shevrin et al. 2013).

Since the psychological content of the unconscious mind cannot be directly accessed or described, the psychoanalytic tradition usually utilizes meaning analysis of its symbolic content expressed in different ways by individuals (e.g., association tests and guided imagery). Nevertheless, it is clear that the source of emotion activation is psychological content within the mind (see Fig. 5). The key distinction between conscious and unconscious emotion is whether the individual can make sense of his or her psychological content (e.g., thoughts and memories) at a conscious level. Conscious emotions are directly available for introspection because individuals can recognize their psychological content, whereas unconscious emotion can only be expressed indirectly through symbolic content that seems irrelevant or irrational but is implicitly associated with previous life events.

Instead of eliciting specific emotions by providing specific situations or preselected stimuli, the psychoanalytic tradition applies a different approach, using techniques that allow subjects to relax and respond freely to the guiding cues or symbolic content in a task. This is probably because many psychoanalytic theories are developed in clinical and therapeutic practice. The psychoanalytic tradition uses the analysis of the symbolic meanings of the data provided by the subject to infer emotional reactions. These data usually are not directly informative about emotions but are a rich source of information that is interpreted by the facilitator on the basis of his or her experience. Measuring emotion in psychoanalytic research is challenging by its nature because the cause of certain emotions is related to personal life events and difficult to justify. 


\section{Models of Emotion}

The above four perspectives represent four different metaphysical hypotheses about the nature of emotion. This section provides a review of models of emotion that are widely accepted and often used in other fields of study. Models of emotion are generalized from emotion theories to allow all kinds of emotional qualities to be classified and conceptualized in a more concrete way. Therefore, models of emotion are considered as direct representations, in that they have been developed as a kind of operational definition that can be applied directly for practical uses, for example, emotion recognition for video game play.

\section{Discrete and Dimensional Models of Emotion}

Psychological models of emotion are mainly based on two views of emotion. The first view holds that emotions are discrete and fundamentally distinct constructs, while the alternative view is that emotions can be represented dimensionally. Some researchers have combined these two perspectives to provide an integrated view (Hamann 2012). It appears that these two models both have advantages and disadvantages in terms of context of use in research.

The discrete model of emotion proposes that there are a limited number of distinct types of emotion, each with characteristic properties (Barrett et al. 2007). This model was originally inspired by one of the evolution-based perspective theories - the basic emotion theory. In accordance with the assumption of this theory, the discrete representation of emotion encompasses a limited number of basic emotions (i.e., happiness, sadness, anger, fear, and disgust) that are universal, biologically inherited, and unique so that each class of discrete emotion has a distinctive physiological and neural profile. Other emotions are either conceptualized as blends of the basic emotions (e.g., contempt is a blend of anger and disgust) or given a different status (e.g., shame is a complex social emotion). The discrete emotion model is mainly characterized by its reliance on linguistic forms. Emotional words such as "anger" are universally understandable references to the corresponding emotional qualities. This aspect of the model has drawn criticism from other researchers for the way in which it confuses the nature of emotion and global terms for labeling emotions (Barrett 2006b). It is also claimed that this model does not make clear predictions on the eliciting conditions for discrete emotions (e.g., the behaviors associated with fear can range from freezing to vigilance to flight, instead of a one-on-one relationship), which has implications for its predictive validity. Moreover, the defining criteria for basic and nonbasic emotions are also unclear.

Unlike the taxonomic approach of the discrete emotion model, the dimensional model of emotion is based on the idea that emotions can be described by several independent and meaningful dimensions (Russell 1979). The current version of dimensional model, known as the circumplex model, makes two primary dimensions: "unpleasant-pleasant" (valence) and "activation-deactivation" (arousal) as 
the core affect. The exact nature of the dimensions and the existence of a circumplex distribution of central emotional states have been debated. Core affect is defined as "the most elementary consciously accessible affective feelings (and their neurophysiological counterparts) that need not be directed at anything" (Russell and Barrett 1999); different prototypical emotional episodes or distinct emotions induced by specific objects can therefore be represented by a combination of the two dimensions. Some researchers have proposed the inclusion of a third, less prominent dimension "in control-dominated" (dominance) (Lang et al. 2008) to distinguish emotions that are in almost the same position in two-dimensional affective space, e.g., anger and fear. There are a number of drawbacks to dimensional theories, such as the absence of attempts to predict theoretically the determinants of differences between emotions and the lack of an explanatory mechanism that predicts response patterning. Moreover, not all researchers agree on the number and nature of dimensions needed in a framework for classifying emotion.

\section{Building Models with Physiological Signals}

Physiological signals are important indirect representations of emotion because they are tightly bound to emotional reactions. Observations of physiological activations in the autonomous nervous system enable researchers to identify and label corresponding psychological states. Psychophysiological instruments have the advantage of being language independent and can be used with individuals from different cultural backgrounds, and individuals do not need to be interrupted with questions during measurement. It has also been suggested that physiological observations could be used to identify unconscious emotional states that are inaccessible through introspective reports (Fairclough 2009). Recent years have seen growing trends toward the application of machine learning techniques for training predictive models of classification based on data obtained from physiological signals. These predictive models can also be considered as a kind of representational model of the emotional responses induced by given classes of affective stimuli, i.e., emotion profiles. However, unlike the discrete model and the dimensional model, which both have meaningful classification rules, a predictive model is a "black box" with parameters that it is difficult to relate to psychological concepts (Fairclough 2009). The classification rate of the predictive model only reflects the homogeneity of the stimuli in each category and the physiological heterogeneity of the groups of responses. Although a considerable body of research has attempted to relate physiological signals to meaningful classification rules such as those used by the discrete and dimensional models, the results obtained of empirical studies still lack consistency, and the variation in results is difficult to explain (Kreibig 2010). What researchers may find more psychologically meaningful is the classification of the affective stimuli that serve to elicit emotion in the unified structure. Many researchers training predictive models strive to achieve a higher recognition rate; developing a well-classified collection of affective stimuli is equally important but has often been overlooked. 
The discrete and the dimensional models have probably gained widespread acceptance because they come close to the folk psychological understanding of emotion, the way in which individuals consciously recognize their own emotional states. However, these two models do not take into account the possibility that emotion may be unconscious. Even if unconscious emotions were to exist, it is unclear that one could simply add one more dimension termed "conscious-unconscious" to the existing dimensional models as it has not yet been confirmed that unconscious emotions and conscious emotions are homologous (e.g., do unconscious joy and conscious joy have the same quality of joy?). Researchers can use physiological signals as the basis for an alternative model of emotion because the modeling algorithms do not have psychological meanings. The model derived from physiological signals can only be interpreted on the basis of the psychological meaning of the classification of the affective stimuli used for emotion elicitation.

\section{Measures of Emotion}

The most common method of measuring emotion in empirical studies is self-report techniques: subjects report their emotional states using introspection. In the case of conscious emotion, most researchers have considered introspective judgments about emotional feelings to be fundamentally valid or correct because it is assumed that human beings are capable of conceptualizing their own emotions in a universally recognized way. Although it is recognized that there are some cultural differences, the expression and interpretation of emotions is considered to be universal at a certain level. Empirical studies have also indicated that self-reports of emotion are likely to be more reliable than other measures although they are limited in some conditions. For example, some people may be unaware of or incapable of reporting their emotional feelings. The Self-Assessment Manikin (SAM) was based on the dimensional model; it was developed by mapping three meaningful dimensions - valence, arousal, and dominance - to Likert scales (Bradley and Lang 1994). Although some researchers have raised concerns about the validity of self-reports of emotions, the SAM scale has been proved effective and is widely used in several research domains. However, self-report techniques are less effective for capturing unconscious emotions because unconscious emotions are unavailable for introspection. To address this, cognitive science has seen an increase in the use of indirect indicators to assess a larger range of human emotions.

As discussed in the section of models of emotion, physiological measures are the most commonly used indirect measures of emotion due to the fact that certain physiological changes coincide with the appearance of specific emotions. Biosensors have proved a powerful tool and have allowed researchers to monitor almost exhaustively the physiological activities of human body in a convenient way. Physiological measurement offers several advantages: emotional experiences can be monitored continuously, physiological signs are not language - or memory dependent, and the measurement process does not interfere with the emotional experience. Using physiological signals also allows researchers to utilize 
computational algorithms to build models of psychological events (Fairclough 2009). A computational model obtained using this approach can be used directly for emotion recognition in practical applications (i.e., make predictions about emotion recognition). Advances in computational algorithms have enabled researchers to keep improving the quality of models based on the same data. Although psychophysiological measurements seem to offer several advantages, some limitations should be noted. Firstly, physiological measurements relate to a broad range of cognitive and affective activities as well as emotional reactions. The extraction of useful information from physiological data is not as intuitive as direct representational methods. Most psychophysiological approaches use supervised classification methods based on introspective judgments about emotional states. In dealing with unconscious emotions, i.e., emotions that are unavailable for introspection, researchers have to consider the risk of falling into epistemological traps when interpreting physiological data (i.e., does the measure index the emotion it is assumed to be related to?). Secondly, previous studies have demonstrated recognition accuracy for various sets of emotions ranging from $30 \%$ to $90 \%$ across different experimental settings (Novak et al. 2012). It appears that physiological responses are influenced not solely by psychological and affective stimuli but by diverse factors such as physical activity, ambient temperature, and individual physiological differences.

Rather than physiological measurements, social psychologists usually look into the changes in behavior, attitude, preference, and decision-making. The social psychological approach has a long history and is widely used in several research domains; it is particularly important in behavioral science. The advantage of this approach is that changes in social psychological measure are intrinsically meaningful, unlike physiological changes. Results are usually self-explanatory and can be interpreted intuitively. The key to this approach is experimental design and inference. Because the social psychological approach focuses specifically on changes, the experimental design must consider the baseline from which changes are to be measured. It is difficult to define a baseline because this leads to a debate on the nature of emotion (e.g., discrete vs. dimensional). Furthermore, because there is not a simple one-to-one causal mapping between behavior and emotion, it is hard to infer the exact emotional cause of a specific behavioral change (e.g., fear and anger both may cause the behavior of fighting). The context and the motivation for the experimental task are also critical factors as they may affect how subjects understand and perform the tasks. This means that data obtained using this approach is usually only valid in the given context and interpretations cannot be generalized.

To obtain data on emotional experiences, the psychoanalytic tradition focuses on the symbolic meaning of the content generated by clients in imagery tasks. Jung (1959) claimed that there is inborn psychological substrate embedded in every human's unconscious mind which cannot be directly accessed or described by the conscious. However, this substrate - which he called archetypes - can be manifested through symbolic content in various forms. The concept of archetypes, although not all psychoanalysts accept it, was the basis of the symbolic tradition in 
psychoanalysis. Within the symbolic tradition, many therapeutic techniques were developed to help clients express their emotional feelings indirectly by guided construction of imagery or association tasks using specific stimulus words, graphics, or objects. For example, the guided affective imagery (GAI) technique uses a narrative script to guide the client and a set of imagery targets such as meadows, trees, and houses as cues to enable clients to indirectly express their emotions (Leuner 1969). The psychoanalytic approach differs from mainstream psychological approaches in that it takes a bottom-up approach. Rather than inducing and recognizing predefined emotions such as joy and sadness, psychoanalysts tend to pose a neutral scenario and observe how people generate symbolic content related to it to express their emotions implicitly. This approach requires that the subject be deeply relaxed (e.g., hypnotized) to facilitate the emergence of unconscious emotions. While the subjective nature of this approach is often criticized by scientific positivists, there is some empirical evidence for the therapeutic efficacy of the psychoanalytical approach (Shedler 2010).

\section{An Integrated Overview of Unconscious Emotion}

According to the above four theoretical perspectives and various representations of emotion, a theoretical overview of emotion research is provided (see Fig. 6). In general, emotion research encompasses three complementary building blocks: theories, models, and measures of emotion. Although these three building blocks share some common ground, they reflect differences in scientific aims and diverse research questions. These three building blocks form a "triangulation" of emotion research (for more discussions about triangulation, see (Moran-Ellis 2006)). They are mutually constraining yet also support each other. When a theoretical perspective is proposed to explain the underlying mechanisms of emotion, researchers can conduct empirical studies to assess the validity of the theory using various measures. On the other hand, data collected from different measures may also be useful for developing new models and theories. While theoretical perspectives concentrate on the ontology and functionality of emotion, models of emotion are intended exhaustively to describe and classify all possible emotions. When it comes to unconscious emotion, it is important to reconsider which theoretical perspective, model, and measures can be used to confirm or refute the existence of this phenomenon and interpret experimental results without falling into epistemological traps.

\section{Research into Unconscious Emotions in Media Content}

Human experience has been promoted to a new research agenda in recent years (Hassenzahl and Tractinsky 2006). Considering media content as information that flows continuously through the audience's mind, the emotional experiences that arise along with the story are of great interests to researchers in related fields. 


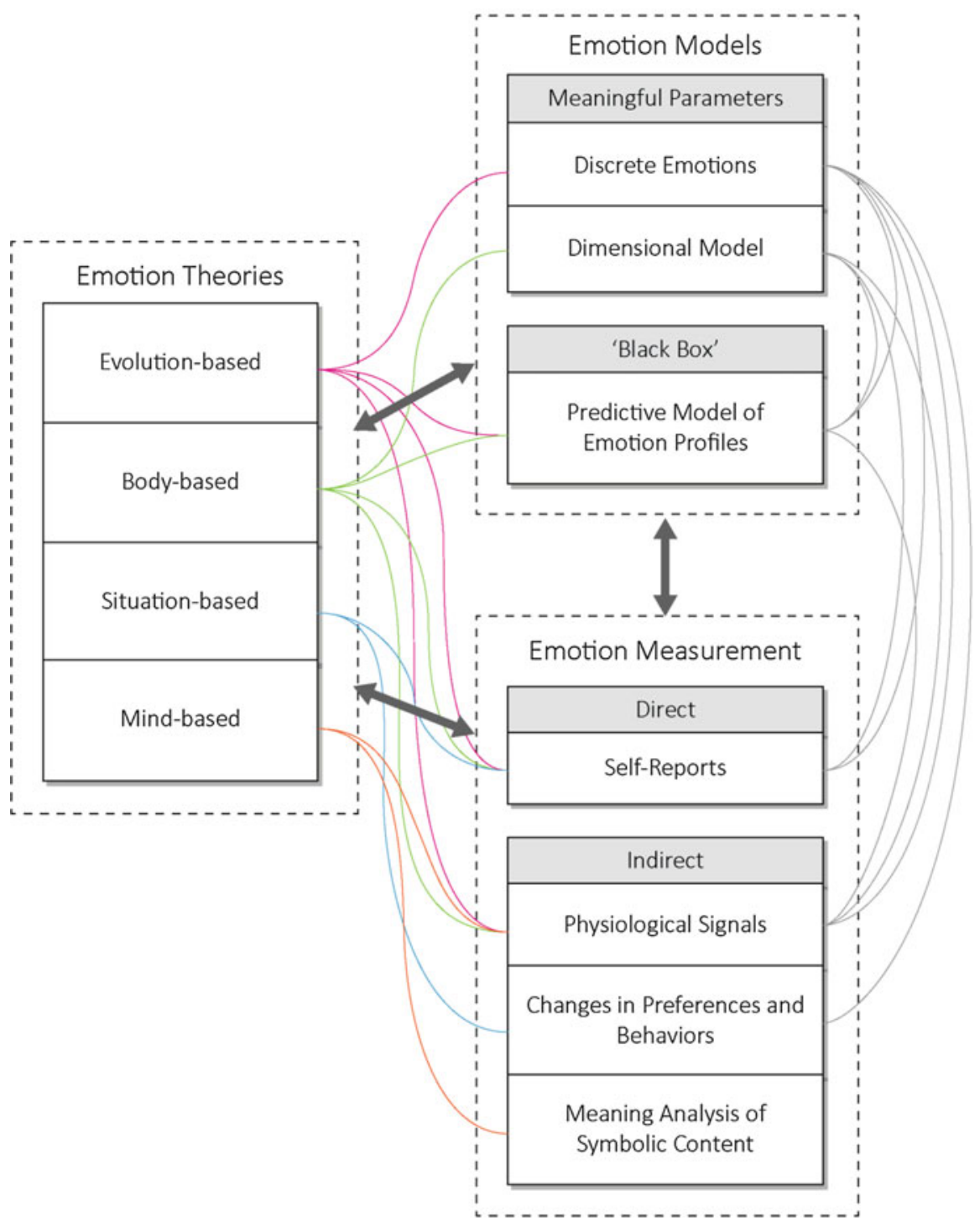

Fig. 6 An integrated overview of emotion research. Thick arrows represent relationships among the three building blocks; thin lines represent approaches that are often used in mainstream research on emotions 


\section{Mapping Media Experience with the Storyline}

Media content stimulates the process of meaning making and the emergence of emotional experiences. A traditional view toward the relationship between the audience and the media is similar to an input-output mechanical process. From this perspective, emotional experiences are nothing more than the outcome of the viewing experience. In recent years, researchers have made considerable progress moving from passive viewing experiences to a multimodal interaction that takes into account affective states of the audience. Various applications of physiological technology are employed to measure and recognize human emotion, so that digital media systems are empowered to react not only to the audience's conscious behaviors but also to their affective states (Chang et al. 2014a). A nice example of new paradigms for applying affective computing in developing digital media is enactive media (Tikka 2010). The theoretical foundation of the idea originates from enactivism in cognitive science, which takes a "groundless" stance that resists the mind-body dualism, and argues that the mind, body, and world are interrelated and interdependent (Varela et al. 1992). Extending enactivism to the cinema experience, cinema is considered as a metaphorical externalization of embodied mind; the audience's mind and the image of the cinema emerge and interplay with and within each other. The audience and the cinema have to be put together into a systematic context that mutually and continuously creates its own meaning.

In the view of enactivism, meaning making is an automated process, and the emotional experiences can be seen as its representation of the interaction between the mind and the story (Chang et al. 2014a). In order to study the relationship between emotion and the media content, the storyline of the media content plays the key role in structuring the whole emotional experience. In the field of mythology, considerable efforts have been devoted to the study of universal storyline across various cultures and religions (Campbell 1973; Propp 1984). This knowledge has been used in screenwriting in today's screenwriting (Field 1984; McKee 1997) and has inspired the making of numerous classic movies, such as the Star War series (Tiffin 1999).

Since the structure of stories is suggested to be universal to a certain level, research into the emotional experiences built upon this storyline is thus a promising direction to proceed. This typical kind of experience was called "archetypal experience," and evidence has suggested that it introduces unique physiological patterns that can be recognized with significance (Ivonin et al. 2015). While the preliminary findings are promising, various genres of movies are yet to be studied. While Hollywood movies are globally well known, Bollywood movies created its own genre and are getting more popular in recent years, especially in Asia and other regions. They provide a great resource for this research direction and might lead to interesting findings. 


\section{Potential Challenges and Promising Approaches}

Regarding the unconscious emotions in media content, some preliminary studies have revealed that specific narratives in media content would induce unique physiological patterns while the audience is unaware of it (Ivonin et al. 2013, 2014, 2015; Chang et al. 2014b). It is promising to explore further with this direction. For example, how could media content influence the audience's attitude, preference, decision-making, and goal setting? Are these effects conscious or unconscious? Numbers of interesting research topics are worthy researching. In order to take the initiative, it is necessary to point out below the potential challenges and outline some promising approaches that could be used in future studies of unconscious emotion in media content.

The first challenge that researchers encounter is the choice of guiding theory or model for future empirical research. As illustrated earlier, the existing theories represent four complementary perspectives about emotion (i.e., the brain, body, situation, and mind). There are considerable overlaps between them, and none can be excluded from a discussion of emotion. Rather than seeing these four theoretical perspectives as conflicting conceptualizations, it is better to consider them as different epistemological approaches to the same phenomena and look for evidence that might lead to new theories and models. As some researchers have pointed out, there are limits to behaviorist explanations of psychological phenomena, and it is time to look for new paradigms in psychology (Kohler 2010). Given that the existing theories of emotion have been well researched, it might be useful to explore human emotions using an integrated approach. Several new ideas have been proposed. For example, an integrated approach based on the "extended mind" considered the environment as an extension of the mind (Clark and Chalmers 1998); thus as individuals change the environment, they are also changing how they experience the world. However, these new theoretical approaches are more like philosophical propositions rather than predictive, explanatory theories. There is a long way to go before they can form the basis of empirical, scientific studies. When it comes to the concept of unconscious emotion, the real challenge is not proving it exists, but providing a good explanatory account of the experimental data to improve current theory in this area.

The other critical challenge is a reevaluation of the basis of measurements of emotion. Because self-reports have been considered the most valid measure of conscious emotion, many studies have regarded the results of introspection as a fundamentally true account of experienced emotions. The accuracy of self-reports relies on their direct relationship with individual emotional experience; all the other measures are indirect and inferential. Unfortunately, these effective measures are unsuitable for assessing unconscious emotion because by definition unconscious emotions only take place below the threshold of introspection. It is epistemologically impossible to assess unconscious emotions through direct measures, which means that there is no easy foundation for models and theories. Since unconscious emotion cannot be accessed directly, it is necessary to use indirect measures. It should also be noted that conscious and unconscious emotions are interrelated and 
may coexist in most cases (Reingold and Merikle 1990). To extract information about unconscious emotion from the overall emotional experience appears to be the most difficult task of all. Recent years have growth in the use of mixed-methods approaches to complex phenomena (Moran-Ellis 2006). It is suggested that researchers should integrate direct and indirect measures in order to investigate phenomena that cannot be measured directly. This would help distinguish conscious and unconscious emotions and perhaps produce less biased interpretations of empirical data.

\section{What Has Been Missing?}

In the overview, it can be seen that many potential theoretical approaches have not yet been used. In particular, mind-based theories have long been marginalized by mainstream scientific studies because the unconscious was, and sometimes still is, treated as a taboo in science. Researchers tend to prefer externally rather than internally oriented explanatory accounts of a given phenomenon, partly because contemporary science is deeply influenced by Newtonian notion of "objectivity" (Chancer 2013). The legacy of the behaviorist tradition has been the emphasis on using observations of human behavior to understand human psychology and a negligence of the concept of mind. Nevertheless, it has recently been asserted that researchers should consider humans outside the machine paradigm (Kohler 2010). The fast, subjective, and irrational aspect of the human mind has been noted by some leading scientists (Kahneman 2003; Kihlstrom 2008). While the best method of investigating unconscious phenomena in empirical studies remains unclear, psychoanalysis might provide a useful starting point for those seeking new paradigms.

The procedures associated with the experimental psychological approach usually encompass both qualitative and quantitative research methods. While emotion recognition has largely relied on quantitative measures such as physiological signals and questionnaires like SAM, inferences and attributions relating to emotion activation can only be understood using qualitative methods. Social psychology looks for explanations in the external world (i.e., situation-based accounts), whereas psychoanalysis searches for answers from the subjective world (i.e., mindbased accounts). Social psychologists often combine quantitative data and qualitative analysis to investigate how social circumstances influence emotional experience. However, little attention has been paid to psychoanalytic studies combining qualitative and quantitative methods. This approach has been missing from contemporary research on emotion, and it seems a promising direction, in particular for researchers who are interested in subjectivity and the unconscious aspects of the human mind. 


\section{Conclusion}

As demonstrated in the overview, many new theoretical approaches have yet to be applied into practice. Research into emotions in media content might even more complex because it is difficult to put media content directly into the context of psychological experiments. Nevertheless, it is a whole new research agenda when taking into account the continuity of media content and the unconscious part of emotional experience. This chapter started with the motivation to research into the relationship between myths and the unconscious. There are numbers of resemblance across different cultures and religions particularly in their myths and stories. Nowadays, these narratives still profoundly influence the human society, shaping the world as it is today. While most scientific research fully concentrates on how media content influences audience at a conscious level, it is also important to look into the irrational part of the media experience that might emerge along with the narratives. This chapter serves as a theoretical basis for researchers who intend to delve into this research direction and new inspirations for inventing new genres of digital media.

\section{Recommended Reading}

M.B. Arnold, Emotion and personality (Columbia University Press, New York, 1960)

J.R. Averill, A constructivist view of emotion, in Emotion: Theory, Research, and Experience, ed. by R. Plutchik, H. Kellerman (Academic, New York, 1980), pp. 305-339

J.A. Bargh, E. Morsella, The unconscious mind. Perspect. Psychol. Sci. 3, $73-79$ (2008)

L.F. Barrett, Are emotions natural kinds? Perspect. Psychol. Sci. 1, 28-58 (2006a). doi:10.1111/ j.1745-6916.2006.00003.x

L.F. Barrett, Solving the emotion paradox: categorization and the experience of emotion. Personal Soc. Psychol. Rev. 10, 20-46 (2006b). doi:10.1207/s15327957pspr1001_2

L.F. Barrett, The future of psychology: connecting mind to brain. Perspect Psychol. Sci. 4, 326-339 (2009). doi:10.1111/j.1745-6924.2009.01134.x

L.F. Barrett, Emotions are real. Emotion 12, $413-429$ (2012). doi:10.1037/a0027555

L.F. Barrett, B. Mesquita, K.N. Ochsner, J.J. Gross, The experience of emotion. Annu. Rev. Psychol. 58, 373-403 (2007). doi:10.1146/annurev.psych.58.110405.085709

A. Bechara, H. Damasio, D. Tranel, A.R. Damasio, The Iowa Gambling Task and the somatic marker hypothesis: some questions and answers. Trends Cogn. Sci. 9, 159-162 (2005). doi:10.1016/j.tics.2005.02.002

B. Bornemann, P. Winkielman, E. van der Meer, Can you feel what you do not see? Using internal feedback to detect briefly presented emotional stimuli. Int. J. Psychophysiol. 85, 116-124 (2012). doi:10.1016/j.ijpsycho.2011.04.007

M.M. Bradley, P.J. Lang, Measuring emotion: the self-assessment manikin and the semantic differential. J. Behav. Ther. Exp. Psychiatry 25, 49-59 (1994)

J. Campbell, The Hero with a Thousand Faces (Princeton University Press, Princeton, 1973)

L.S. Chancer, Sociology, psychoanalysis, and marginalization: unconscious defenses and disciplinary interests. Soc. Forum 28, 452-468 (2013). doi:10.1111/socf.12033

H.-M. Chang, L. Ivonin, M. Díaz et al., From mythology to psychology: identifying archetypal symbols in movies. Technoetic Arts 11, 99-113 (2013). doi:10.1386/tear.11.2.99_1 
H.-M. Chang, L. Ivonin, M. Diaz et al., Enacting archetypes in movies: grounding the unconscious mind in emotion-driven media. Digit. Creat. 26, 154-173 (2014a). doi:10.1080/ 14626268.2014 .939985

H.-M. Chang, L. Ivonin, M. Diaz et al., Unspoken emotions in movies. Informatik-Spektrum 37, 539-546 (2014b). doi:10.1007/s00287-014-0823-3

A. Clark, D.J. Chalmers, The extended mind. Analysis 58, 10-23 (1998)

A.R. Damasio, The somatic marker hypothesis and the possible functions of the prefrontal cortex. Philos. Trans. R. Soc. Lond. B Biol. Sci. 351, 1413-1420 (1996). doi:10.1098/rstb.1996.0125

A. Dijksterhuis, Think different: the merits of unconscious thought in preference development and decision making. J. Pers. Soc. Psychol. 87, 586-598 (2004). doi:10.1037/0022-3514.87.5.586

A. Dundes, The flood as male myth of creation, in The Flood Myth, ed. by A. Dundes (University of California Press, London, 1988), pp. 167-182

S.H. Fairclough, Fundamentals of physiological computing. Interact. Comput. 21, 133-145 (2009). doi:10.1016/j.intcom.2008.10.011

S. Field, Screenplay: The Foundations of Screenwriting (Dell Publishing, New York, 1984)

S. Freud, The unconscious. J. Nerv. Ment. Dis. 56, 291-294 (1922)

N.H. Frijda, The laws of emotion. Am. Psychol. 43, 349-358 (1988). doi:10.1037/0003066X.43.5.349

M. Gendron, L.F. Barrett, Reconstructing the past: a century of ideas about emotion in psychology. Emot. Rev. 1, 316-339 (2009). doi:10.1177/1754073909338877

G. Gigerenzer, Gut Feelings: The Intelligence of the Unconscious (Penguin Group, New York, 2007)

S. Hamann, Mapping discrete and dimensional emotions onto the brain: controversies and consensus. Trends Cogn. Sci. 16, 458-466 (2012). doi:10.1016/j.tics.2012.07.006

M. Hassenzahl, N. Tractinsky, User experience - a research agenda. Behav. Inf. Technol. 25, 91-97 (2006). doi:10.1080/01449290500330331

L. Ivonin, H.-M. Chang, W. Chen, M. Rauterberg, Unconscious emotions: quantifying and logging something we are not aware of. Pers. Ubiquitous Comput. 17, 663-673 (2013). doi:10.1007/ s00779-012-0514-5

L. Ivonin, H.-H. Chang, M. Díaz, et al., Beyond cognition and affect: sensing the unconscious. Behav. Infect. Technol. 1-19 (2014). doi:10.1080/0144929X.2014.912353

L. Ivonin, H.-M. Chang, M. Diaz et al., Traces of archetypal experiences in introspective reports and physiological responses. PLoS One 10, e0124519 (2015). doi:10.1371/journal. pone. 0124519

C.E. Izard, Emotion theory and research: highlights, unanswered questions, and emerging issues. Annu. Rev. Psychol. 60, 1-25 (2009). doi:10.1146/annurev.psych.60.110707.163539

W. James, What is an emotion? Mind 9, 188-205 (1884)

C.G. Jung, The Archetypes and the Collective Unconscious (Princeton University Press, Princeton, 1959)

D. Kahneman, Maps of bounded rationality: psychology for behavioral economics. Am. Econ. Rev. 93, 1449-1475 (2003). doi:10.1257/000282803322655392

J.F. Kihlstrom, The psychological unconscious, in Handbook of Personality: Theory and Research, ed. by O. John, R. Robins, L. Pervin, 3rd edn. (Guilford Press, New York, 2008), pp. 583-602

J.F. Kihlstrom, Unconscious cognition, in Encyclopedia of Consciousness, ed. by W.P. Banks (Academic, Oxford, 2009), pp. 411-421

J.F. Kihlstrom, S. Mulvaney, B.A. Tobias, I.P. Tobis, The emotional unconscious, in Counterpoints: Cognition and Emotion, ed. by E. Eich, J.F. Kihlstrom, G.H. Bower et al. (Oxford University Press, New York, 2000), pp. 30-86

G.S. Kirk, The Nature of Greek Myths (Penguin, New York, 1975)

A. Kohler, To think human out of the machine paradigm: Homo Ex Machina. Integr. Psychol. Behav. Sci. 44, 39-57 (2010). doi:10.1007/s12124-010-9113-z 
S.D. Kreibig, Autonomic nervous system activity in emotion: a review. Biol. Psychol. 84, 394-421 (2010). doi:10.1016/j.biopsycho.2010.03.010

P.J. Lang, Emotion and motivation: toward consensus definitions and a common research purpose. Emot. Rev. 2, 229-233 (2010). doi:10.1177/1754073910361984

P.L. Lang, M.M. Bradley, B.N. Cuthbert, International affective picture system (IAPS): affective ratings of pictures and instruction manual. In: Technical Report A-8. University of Florida, Gainesville, pp. 1-12 (2008)

H. Leuner, Guided affective imagery (GAI): a method of intensive psychotherapy. Am. J. Psychother. 23, 4-22 (1969)

B. Malinowski, Myth in primitive psychology, in Magic, Science and Religion and Other Essays (Doubleday, New York, 1954), pp. 93-148

J.G. Manis, B.N. Meltzer, Symbolic Interaction: A Reader in Social Psychology, 3rd edn. (Allyn and Bacon, Boston, 1978)

R.A. Mar, K. Oatley, The function of fiction is the abstraction and simulation of social experience. Perspect. Psychol. Sci. 3, 173-192 (2008). doi:10.1111/j.1745-6924.2008.00073.x

R. May, The Cry for Myth (W. W. Norton, New York, 1991)

R. McKee, Story: Substance, Structure, Style, and the Principles of Screenwriting (Harper-Collins Publishers, New York, 1997)

J. Moran-Ellis, Triangulation and integration: processes, claims and implications. Qual. Res. 6, 45-59 (2006). doi:10.1177/1468794106058870

D. Novak, M. Mihelj, M. Munih, A survey of methods for data fusion and system adaptation using autonomic nervous system responses in physiological computing. Interact. Comput. 24, 154-172 (2012). doi:10.1016/j.intcom.2012.04.003

K. Oatley, Why fiction may be twice as true as fact: Fiction as cognitive and emotional simulation. Rev. Gen. Psychol. 3, 101-117 (1999). doi:10.1037/1089-2680.3.2.101

A. Öhman, Face the beast and fear the face: Animal and social fears as prototypes for evolutionary analyses of emotion. Psychophysiology 23, 123-145 (1986). doi:10.1111/j.1469-8986.1986. tb00608.x

V. Propp, Theory and History of Folklore (University of Minnesota Press, Minneapolis, 1984)

M. Rauterberg, The three phases of life: an inter-cultural perspective. In: Proceedings of the 2nd International Conference on Culture and Computing. IEEE Computer Society, Los Alamitos, pp. 80-85 (2011)

E.M. Reingold, P.M. Merikle, On the inter-relatedness of theory and measurement in the study of unconscious processes. Mind Lang. 5, 9-28 (1990)

J. Rottenberg, R.D. Ray, J.J. Gross, Emotion elicitation using films, in Handbook of Emotion Elicitation and Assessment, ed. by J.A. Coan, J.J.B. Allen (Oxford University Press, Oxford, 2007), pp. 9-28

J.A. Russell, Affective space is bipolar. J. Pers. Soc. Psychol. 37, 345-356 (1979). doi:10.1037/ 0022-3514.37.3.345

J.A. Russell, L.F. Barrett, Core affect, prototypical emotional episodes, and other things called emotion: dissecting the elephant. J. Pers. Soc. Psychol. 76, 805-819 (1999)

S. Schachter, J. Singer, Cognitive, social, and physiological determinants of emotional state. Psychol. Rev. 69, 379-399 (1962). doi:10.1037/h0046234

K.R. Scherer, What are emotions? And how can they be measured? Soc. Sci. Inf. 44, 695-729 (2005). doi:10.1177/0539018405058216

J. Shedler, The efficacy of psychodynamic psychotherapy. Am. Psychol. 65, 98-109 (2010). doi: $10.1037 / \mathrm{a} 0018378$

H. Shevrin, M. Snodgrass, L.A.W. Brakel et al., Subliminal unconscious conflict alpha power inhibits supraliminal conscious symptom experience. Front. Hum. Neurosci. 7, 544 (2013). doi:10.3389/fnhum.2013.00544

J. Tiffin, Digitally remythicised: Star Wars, modern popular mythology, and Madam and Eve. J. Lit. Stud. 15, 66-80 (1999) 
P. Tikka, Enactive media - generalising from enactive cinema. Digit. Creat. 21, 205-214 (2010). doi:10.1080/14626268.2011.550028

F.J. Varela, E.T. Thompson, E. Rosch, The Embodied Mind: Cognitive Science and Human Experience (MIT Press, Cambridge, MA, 1992)

T.D. Wilson, Knowing when to ask: introspection and the adaptive unconscious. J. Conscious Stud. 10, 131-140 (2003)

T.D. Wilson, Strangers to Ourselves: Discovering the Adaptive Unconscious (Belknap, Cambridge, MA, 2004)

P. Winkielman, K.C. Berridge, J.L. Wilbarger, Unconscious affective reactions to masked happy versus angry faces influence consumption behavior and judgments of value. Pers. Soc. Psychol. Bull. 31, 121-135 (2005). doi:10.1177/0146167204271309

Y. Zemack-Rugar, J.R. Bettman, G.J. Fitzsimons, The effects of nonconsciously priming emotion concepts on behavior. J. Pers. Soc. Psychol. 93, 927-939 (2007). doi:10.1037/00223514.93.6.927 